\title{
DESIGUALDADES ESPACIALES DE HABITABILIDAD EN CIUDADES PATRIMONIALES. APLICACIÓN DE INDICADORES DE SEGUIMIENTO A LAS CIUDADES DE SAN GABRIEL Y SANGOLQUI (ECUADOR)
}

\author{
Elena Usobiaga Ferrer \\ Tecnalia Research \& Innovation \\ elena.usobiaga@tecnalia.com \\ Olga de Cos Guerra \\ Departamento de Geografía, Urbanismo y Ordenación del Territorio. Universidad de Cantabria \\ olga.decos@unican.es

\section{Ángela de Meer Lecha-Marzo} \\ Departamento de Geografía, Urbanismo y Ordenación del Territorio. Universidad de Cantabria \\ angela.meer@unican.es
}

\section{RESUMEN}

Este artículo, contextualizado en ciudades de gran valor patrimonial en Ecuador, plantea la necesidad de compatibilizar la importante protección del patrimonio y la imprescindible garantía de unas condiciones de habitabilidad adecuadas. Para ello se desarrolla una metodología de estudio de la habitabilidad que permita determinar las mejores variables o indicadores para su comprensión y posterior tratamiento urbanístico. Con ello, se da respuesta al conflicto existente entre conservación e intervención urbanística para la mejora de las condiciones de vida de la población.

Palabras clave: ciudad patrimonial, habitabilidad, SIG, trabajo de campo, indicadores, Ecuador, inhabitabilidad.

Fecha de recepción: enero 2016.

Fecha de aceptación: noviembre 2016. 


\section{ABSTRACT}

This article, contextualized in Ecuadorian cities of great historical value, raises the need to reconcile the protection of cultural heritage with the essential guarantee of habitability adequate conditions. To do this, a methodology for habitability study is developed. It serves to select the most suitable variables or indicators for habitability understanding and its subsequent urban treatment. In doing so, it answer to the conflict among heritage preservation and urban intervention for the improvement of population living conditions.

Keywords: heritage city, habitability, GIS, field work, indicators, Ecuador, inhabitability.

\section{INTRODUCCIÓN}

Este artículo se centra en definir la metodología más adecuada para tratar el complejo tema de la habitabilidad en las ciudades patrimoniales, una problemática de gestión urbanística de gran interés en proyectos de intervención en dichos ámbitos urbanos. El estudio analiza dos ciudades ecuatorianas, San Gabriel y Sangoquí, con el objetivo de determinar la metodología para abarcar la complejidad del análisis de la habitabilidad.

Con este fin, se realiza un planteamiento multiescala basado en Sistemas de Información Geográfica (SIG) a través de la combinación de herramientas de análisis espacial y social. Esta metodología permite un acercamiento más preciso al problema analizado (la habitabilidad) dentro de su propio contexto -las ciudades de San Gabriel y Sangolquí-, permitiendo así seleccionar los indicadores que mejor representen la habitabilidad para cada ciudad. En este artículo se presenta el proceso de análisis seguido en ambas ciudades, haciendo una comparativa de los resultados obtenidos para cada una de ellas.

La metodología presentada forma parte de la tesis doctoral "La habitabilidad como enfoque de la gestión patrimonial en Ecuador. Propuesta de modelo para su gestión y seguimiento" que fue defendida exitosamente en diciembre $2013^{1}$. Su objetivo era el diseño de un modelo

1 Esta tesis doctoral parte de una línea de investigación comenzada en el centro de investigación aplicada Tecnalia Research \& Innovation en 2007 en la que se parte de la base del trabajo de la UNESCO "IAPH, 1999: Indicadores para la evaluación del estado de conservación de ciudades históricas" (Editorial Comares-Granada) y se realiza una reflexión y una propuesta de sistema de indicadores para el seguimiento de la gestión de ciudades históricas. Esta línea sirve de base de diversos trabajos de reflexión teórico-metodológica enfocados al desarrollo y definición de indicadores de seguimiento entre los que se encuentra el desarrollo de esta investigación. Con estos antecedentes, finalmente el origen y ejecución de la tesis doctoral se enmarca dentro un proyecto de cooperación al desarrollo, titulado "Fortalecimiento de las capacidades locales para la mejora de la calidad de vida en las ciudades históricas de Ecuador" cofinanciado por el Gobierno Vasco, bajo la convocatoria FOCAD 2009. Este proyecto se desarrolla en Ecuador, en colaboración con el Instituto Nacional de Patrimonio Cultural, la Municipalidad de Montufar (Región del Carchi), el Programa ART de las Naciones Unidas para el Desarrollo en Ecuador y Tecnalia Research \& Innovation (País Vasco).

Desde el punto de vista académico, la tesis doctoral dirigida por las doctoras Olga de Cos y Ángela de Meer (pertenecientes al Departamento de Geografía, urbanismo y ordenación del territorio de la Universidad de Cantabria.) se enmarca en el Master de Patrimonio Histórico y Territorial de la Universidad de Cantabria, y supone un punto de conexión entre las dos especialidades de la formación impartida, la histórica y la territorial, planteando desde el ámbito de la gestión del patrimonio, la integración de los diferentes conocimientos y herramientas estudiados durante el desarrollo de las asignaturas del master. Así mismo, esta tesis supone una profundización en la relación entre la sociedad y el patrimonio cultural, a través de la intervención en el mismo. 
metodológico para definir sistemas de indicadores y su posterior aplicación al análisis de la habitabilidad en el contexto ecuatoriano. El diseño de un sistema de indicadores, tal como se puede ver en la Figura 1, se concibe como un proceso, que partiendo de una definición teórica basada en el análisis del fenómeno que se quiere medir (la habitabilidad) y en la casuística de dos ciudades de estudio, va evolucionando con cada herramienta implementada, hasta ir configurando un sistema de indicadores más preciso e interpretable y ajustado a cada caso de estudio.

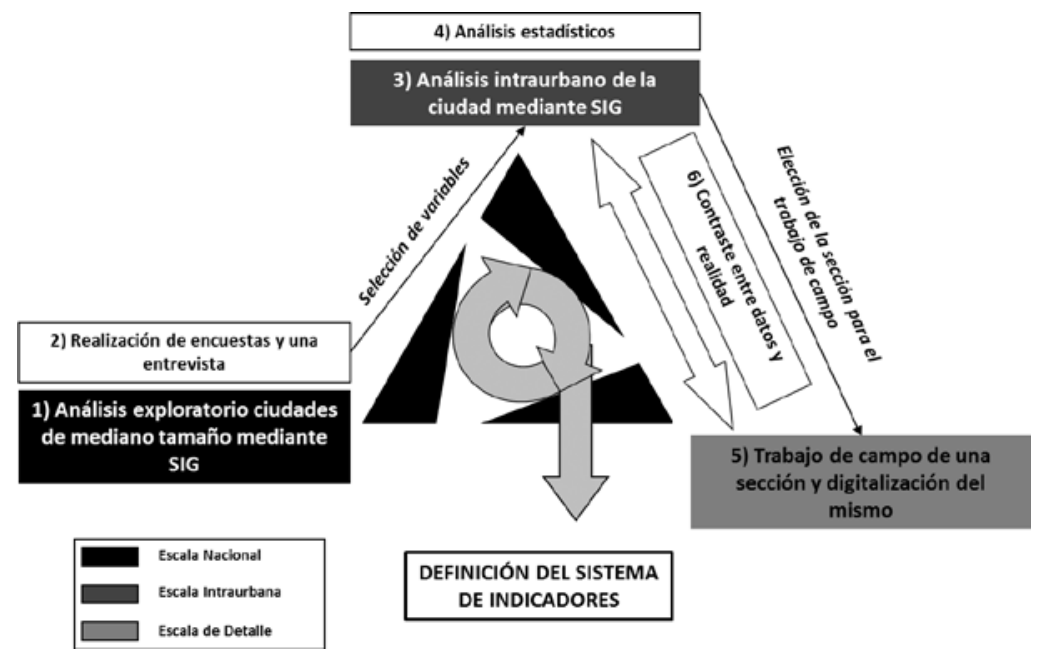

Fuentes: USOBIAGA, E. 2012:527.

\section{COMPRENDIENDO LA HABITABILIDAD COMO UN CONCEPTO MÚLTIPLE Y DINÁMICO}

El ser humano desde siempre se ha preocupado de ir modelando y adaptando su hábitat con el fin de mejorar su bienestar (Solanas, 2010), a medida que avanzaba su conocimiento y medios técnicos. Pero no es hasta el siglo XIX con la aparición de diferentes epidemias de cólera en Europa, que aparecen de manera legislada los requisitos higiénicos mínimos que deben cumplir las viviendas como manera de combatir la aparición y propagación de enfermedades. Estos requisitos, en un principio relacionados con la iluminación y ventilación de los espacios, fueron ampliando su espectro desde la reforma interior de las viviendas hacia la reforma de las ciudades con la instalación de redes de saneamiento, la introducción de zonas verdes, los proyectos de apertura de grandes arterias o ampliación del viario tradicional.

A finales del siglo XIX, la gestión de la salud urbana, condición básica de la habitabilidad, se apoyaba, en lo que a vivienda se refiere, en los avances técnicos como las redes de saneamiento, el acceso generalizado al agua potable o la limpieza de calles.

En su concepción más actual, tal como precisa la Real Academia Española de la Lengua, la habitabilidad es: "Cualidad de habitable, y en particular la que, con arreglo a determina- 
das normas legales, tiene un local o una vivienda"; por lo que tiene una asociación directa con la normativa legal, la cual define los estándares que una vivienda debe cumplir para ser considerada habitable. Detrás de su definición legal se encuentran conceptos, como la salud o el confort, que han ido perfilando las condiciones o requisitos a cumplir y que han visto su reflejo en la legislación vigente.

La habitabilidad desde la salud considera la vivienda como un "determinante de salud" del individuo (Solanas, 2010), y analiza los riesgos que pueden producirse en la salud a nivel tanto físico como psicológico.

La habitabilidad desde el confort o la comodidad "se define como el conjunto de condiciones ambientales aceptada como suficiente por parte de los usuarios para llevar a cabo actividades ordinarias, y por lo general, se limita a las condiciones relativas a los aspectos higro-térmico, acústico y visual, además de estar circunscrita a la escala de la vivienda" (Dálençon, et. al., 2008).

Tabla 1

PRINCIPALES CARACTERISTIICAS DE LOS ENFOQUES DE LA HABITABILIDAD ANALIZADOS

\begin{tabular}{|c|c|c|c|}
\hline $\begin{array}{l}\text { Fin } \\
\text { perseguido }\end{array}$ & Objetivos & Escalas & Factores \\
\hline Salud & $\begin{array}{l}\text { Salud individual y } \\
\text { comunitaria (vivienda } \\
\text { saludable). }\end{array}$ & $\begin{array}{l}\text {-Vivienda } \\
\text {-Entorno } \\
\text { inmediato }\end{array}$ & $\begin{array}{l}\text {-Prestación funcional } \\
\text {-Adecuado desarrollo físico, social } \\
\text { y mental de las condiciones de } \\
\text { salud } \\
\text {-Seguridad } \\
\text {-Higiene } \\
\text {-Comodidad } \\
\text {-Privacidad }\end{array}$ \\
\hline Derechos & $\begin{array}{l}\text { Garantizar unas } \\
\text { condiciones mínimas de } \\
\text { habitabilidad. }\end{array}$ & $\begin{array}{l}\text {-Vivienda } \\
\text {-Entorno } \\
\text { inmediato } \\
\text {-Conjunto } \\
\text { habitacional }\end{array}$ & $\begin{array}{l}\text {-Físico espacial } \\
\text {-Psico social } \\
\text {-Acústico } \\
\text {-Térmico } \\
\text {-Lumínico } \\
\text {-Higiene } \\
\text {-Fuego y accidentes }\end{array}$ \\
\hline Confort & $\begin{array}{l}\text { Incrementar las } \\
\text { condiciones de } \\
\text { habitabilidad }\end{array}$ & -Vivienda & $\begin{array}{l}\text {-Espacios funcionales } \\
\text {-Instalaciones y suministros }\end{array}$ \\
\hline Conservación & $\begin{array}{l}\text { Conservar el carácter } \\
\text { patrimonial del bien } \\
\text { mediante intervenciones } \\
\text { respetuosas de mínimo } \\
\text { impacto sobre el entorno } \\
\text { urbano y natural. }\end{array}$ & $\begin{array}{l}\text {-Vivienda } \\
\text {-Entorno urbano } \\
\text {-Entorno natural }\end{array}$ & $\begin{array}{l}\text {-Materiales } \\
\text {-Estructuras } \\
\text {-Estética (percepción visual) } \\
\text {-Carácter patrimonial } \\
\text {-Tipologías }\end{array}$ \\
\hline
\end{tabular}

Fuente: elaboración propia.

2 Real Academia Española (C) Todos los derechos reservados http://www.rae.es/rae.html [Consultado en Mayo 2013]. 
Hoy en día, más allá de lo legislativo, se tiende a considerar la habitabilidad desde un enfoque cada vez más amplio, que abarca diferentes escalas "vivienda, entorno inmediato, conjunto habitacional" y multitud de factores a tener en cuenta, tales como, físico-espaciales, psico-sociales, térmicos, acústicos, lumínicos, de seguridad, etc. La habitabilidad se redefine y analiza en cada proyecto en función de los objetivos y fines buscados.

Así, los diferentes enfoques de habitabilidad varían en función del fin perseguido tal y como se mostró en la Tabla 1, en la que se presentaron los cuatro más destacados y sus principales características: objetivo al que atienden, escala/s de trabajo y factores que consideran como parte del fenómeno de habitabilidad.

\section{II.1. La habitabilidad desde la salud}

Esta perspectiva está fundamentalmente centrada en el individuo, aunque dado su origen vinculado a la prevención contra enfermedades contagiosas, se considera asimismo asociada a la salud pública y, por tanto, a la sociedad. El enfoque analiza los riesgos que una vivienda inadecuada tiene sobre la salud del individuo, considerando la vivienda como un "determinante de salud" de la persona (Dálençon, et. al., 2008).

En 1990 la Organización Mundial de la Salud (OMS) redacta el documento "Principios de higiene de la vivienda" en el que se detallan once principios fundamentales 3 que relacionan las características de la vivienda y su entorno con la salud de sus habitantes. Este documento, que se ocupa principalmente de la situación de los países en desarrollo, destaca los factores habitacionales que influyen en la salud, basándose en la existencia probada de vínculos entre las malas condiciones de vivienda y un mayor riesgo de accidentes, enfermedades y fallecimientos.

La "Estrategia de vivienda saludable", definida en 2006 por la OMS y la Organización Panamericana de Salud (OPS), hace una revisión del papel central que la vivienda tiene en la vida del individuo tanto para su salud como para su desarrollo personal (desarrollo psicológico y social), así como para su acceso a su centro de trabajo o estudios y diversos servicios imprescindibles para la vida y el disfrute diario, que contribuyen a la salud del individuo. Así pues, se hace referencia a la importancia del entorno de la vivienda como un importante factor de salud, desde aspectos como la seguridad o el acceso a redes de transporte, comunicación, infraestructuras de saneamiento, de agua potable, etc.

Además, se incorporan algunas reflexiones importantes como el hecho de que la vivienda no es sólo una estructura física, sino que se le debe añadir el concepto de uso que el residente da a la vivienda, y la idea de que "el concepto de vivienda saludable no se limita al diseño de la misma, sino que está condicionado también por su localización y construcción, y que se extiende luego a su uso y mantenimiento" (OPS, 2006: 14).

3 Principios relativos a las necesidades sanitarias: 1) Protección contra las enfermedades transmisibles; 2) Protección contra los traumatismos, las intoxicaciones y las enfermedades crónicas; 3) Reducción al mínimo de los factores de estrés psicológicos y sociales; 4) Mejora del entorno habitacional; 5) Uso adecuado de la vivienda; 6) Protección de las poblaciones especialmente expuestas; y Principios relativos a la acción sanitaria: 7) Propaganda de la salud; 8) Políticas económicas y sociales; 9) Acción intersectorial para la planificación y la gestión del desarro1lo; 10) Educación para una vivienda salubre; 11) Cooperación y autoayuda comunitarias. 


\section{II.2. La habitabilidad desde un enfoque de derechos}

Este enfoque se refiere a la importancia de la vivienda como derecho social, como el derecho universal de disponer de vivienda, tal y como se refleja en el artículo 25.1 de la Declaración de Derechos Humanos (ONU, 1948).

El derecho a vivienda, recogido por las constituciones y legislaciones de muchos países añade en muchos casos el derecho a una vivienda "digna y adecuada" o una vivienda saludable como se ha planteado en el enfoque anterior. Así, existe una trayectoria a nivel mundial con respecto al derecho a vivienda y, en especial, a una vivienda adecuada en la constitución del programa HABITAT de las Naciones Unidas, con dos conferencias centradas en este hecho: las conferencias de la ONU sobre los Asentamientos Humanos (I y II) celebradas en Vancouver en 1976 y en Estambul en 1996. Éstas dan como resultado la "Declaración de Vancouver de Asentamientos Humanos" y la "Declaración de Estambul sobre los Asentamientos Humanos"; ambas sirven para concienciar a los diferentes Estados sobre el problema de la vivienda y buscar su compromiso en la mejora de las condiciones de los asentamientos humanos, así como para presentar las acciones del programa HABITAT de las Naciones Unidas en esa misma dirección.

Desde esta perspectiva se valora la vivienda desde su diseño y funcionalidad, estableciéndose unos criterios mínimos de habitabilidad legislados o enumerados en documentos de referencia internacional, con el objeto de lograr unos requisitos mínimos para considerar habitable una vivienda y asegurar la universalización de este derecho.

\section{II.3. La habitabilidad desde el confort y la conservación}

La consideración de la habitabilidad desde la conservación proviene del interés de la arquitectura por el diseño de la vivienda y sus funciones. El principal y único beneficiario al que alude esta perspectiva es el individuo, y su principal objetivo es lograr alcanzar los requisitos mínimos de habitabilidad, que garanticen que una vivienda sea confortable o cómoda para el individuo que vive en ella.

El confort, tal y como señala Briz (2010: 2) “es una cuestión subjetiva, cultural y evolutiva, que en el SXVIII se asociaba a lo privado, lo doméstico, la intimidad; en el S XIX venia determinado por la intervención de lo mecánico: La luz, el calor, la ventilación; hoy puede hablarse de eficiencia, de comodidad... conceptos que se añaden a los ya sedimentados a lo largo del tiempo".

En definitiva, se persigue la evaluación de las prestaciones de la vivienda con el objetivo de minimizar las posibles carencias para mejorar la calidad y comodidad del individuo.

Por otro lado, el enfoque que alude a la conservación, se centra principalmente en la conservación del edificio desde su vertiente patrimonial; aunque, en ocasiones se alude también a la sostenibilidad como una forma de conservación de su entorno o del medio, a través de la no alteración del paisaje o el aprovechamiento de la edificación ya existente para evitar así costes adicionales de recursos naturales y energéticos.

Desde la conservación se considera la adaptación de las viviendas a las necesidades actuales de habitabilidad. El confort, la accesibilidad, la funcionalidad, la sostenibilidad o la flexibilidad son algunos ejemplos de estas demandas, tendencias que están adoptando el 
diseño de nuevas viviendas y deben ser incorporadas también a las viviendas pre-existentes para garantizar su "valor de uso". La imposibilidad de adaptar una vivienda a las necesidades actuales (debido, por ejemplo, a las restricciones impuestas por su valor histórico o patrimonial) conllevan en muchos casos el abandono de la vivienda por parte de sus propietarios, y ésta, al perder el mantenimiento y cuidado derivado de su uso, termina deteriorándose, generando una pérdida patrimonial mayor que la que podría haber supuesto su adaptación.

Con el fin de salvar este problema, existen actualmente investigaciones en la línea de adaptar la vivienda histórica a las necesidades de eficiencia energética, accesibilidad, etc. de manera respetuosa con su valor patrimonial.

En suma, hoy en día se tiende a considerar la habitabilidad desde un enfoque cada vez más amplio e integrado, que abarca diferentes enfoques, distintas escalas -desde la vivienda al entorno inmediato e incluso hasta el conjunto habitacional (Dálençon, et. al., 2008)- y multitud de factores (físico-espaciales, psico-sociales, térmicos, acústicos, lumínicos, de seguridad, etc.). La habitabilidad se redefine y analiza en cada proyecto en función de los objetivos y fines buscados.

\section{II.4. El desarrollo socio-económico como factor determinante en el objeto de la habitabilidad}

Los diferentes enfoques de habitabilidad parecen responder a variadas condiciones socioeconómicas, o a distintas necesidades, pues si se toma como referencia la teoría de la pirámide de necesidades de Maslow que identifica la existencia de una jerarquía de necesidades humanas y defiende que conforme se satisfacen las más básicas, los seres humanos desarrollan necesidades y deseos más elevados, esta misma lógica podría aplicarse a la habitabilidad, tal como se hace en la Figura 2 en la que se jerarquizan las necesidades de habitabilidad en contraste con la jerarquía de necesidades de Maslow.

Figura 2

CONTRASTE DE LA JERARQUIIA DE NECESIDADES DE HABITABILIDAD CON LA JERARQUIIA DE NECESIDADES DE MASLOW

Jerarquía de necesidades de habitabilidad
Jerarquía de necesidades de

Maslow

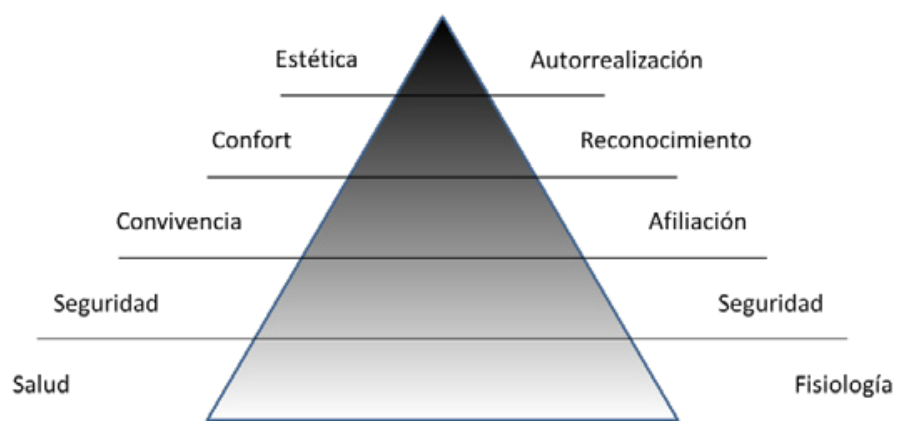

Fuente: elaboración propia. 
Esta jerarquía nos permite comprender por qué en países con menor desarrollo socioeconómico o áreas más desfavorecidas la habitabilidad se centra más en aspectos de salud, seguridad o convivencia; mientras que en los que ostentan un cierto desarrollo y tienen ya estas necesidades resueltas, la habitabilidad se centra más en el confort, la estética o la conservación.

Este aspecto es importante para comprender que la habitabilidad es un concepto y un objeto de estudio que tiene diferentes gradaciones y, por tanto, su análisis se debe considerar desde su base, y en la medida en que los problemas más básicos estén resueltos ir considerando los aspectos siguientes en la jerarquía. Esto obliga a considerar la situación particular en que se encuentra una ciudad antes de tratar de replicar modelos o análisis preexistentes.

\section{METODOLOGÍA GENERAL: LA INTEGRACIÓN DE VARIABLES, TÉCNICAS Y HERRAMIEN- TAS EN EL ESTUDIO DE LA HABITABILIDAD}

El proceso metodológico seguido en este estudio está basado en una integración de técnicas de análisis de datos provenientes de diferentes campos disciplinares, como son: el análisis cartográfico a través de Sistemas de Información Geográfica, el análisis estadístico mediante técnicas de análisis multivariante (factorial y clúster), y la incorporación del criterio experto a través de herramientas de análisis social.

A continuación se presentan las diferentes técnicas empleadas para el análisis de la habitabilidad (Figura 3), que van sucesivamente transformando las variables empleadas para el análisis a través de los resultados obtenidos de la implementación de cada una de las técnicas empleadas.

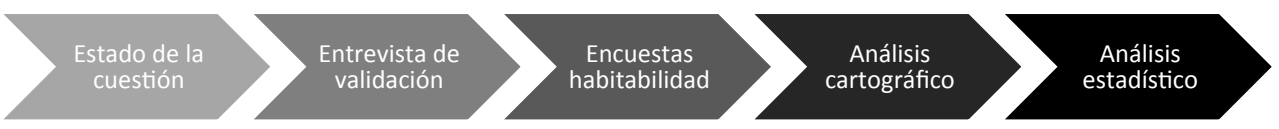

Fuente: elaboración propia.

En primer lugar, se parte de una reflexión sobre el estado de la cuestión de la habitabilidad y el contexto ecuatoriano. Este estado de la cuestión permite realizar una primera selección de las dimensiones y variables a considerar bajo el concepto de la "habitabilidad".

Realizado un primer análisis de la habitabilidad en Ecuador y sus posibles dimensiones, este primer enfoque se valida y completa mediante una entrevista semi-estructurada a la Directora de Planificación del Instituto Nacional de Patrimonio Cultural del Ecuador. Con este primer enfoque validado, se realizan encuestas a técnicos municipales de las ciudades analizadas con el fin de detectar los principales problemas de habitabilidad de las mismas. Este paso permite realizar una selección de variables específicas para cada ciudad, que es posteriormente analizada mediante cartografía estadística y análisis estadísticos, siendo modificada por los resultados de cada uno de estos análisis. 
Estos últimos análisis (el cartográfico y el estadístico) sirven para analizar los patrones espaciales de las variables analizadas, su relevancia en ambas ciudades, seleccionar las variables más significativas en la medición del fenómeno y la interrelación existente entre estas variables. Así mismo, permiten descartar aquellas variables que no son significativas en la medición del fenómeno o aquellas que duplican mediciones, generando una selección depurada para cada ciudad que permite clasificar las áreas de la ciudad (secciones censales en este caso) en función de su grado de vulnerabilidad en relación a la habitabilidad. Es importante señalar que los análisis cartográfico y estadístico se realizan a escala de sección censal debido a que es ésta la unidad de mayor desagregación para la que existen datos públicos, homogéneos y fiables de población, entorno y vivienda. Se busca con ello garantizar la posibilidad de replicar la metodología en otras ciudades del país e incluso en otras ciudades latinoamericanas de características similares, así como diseñar un sistema de indicadores que sea aplicable y actualizable al menor coste posible.

\section{III.1. Concretando el concepto de habitabilidad}

Vista la diversidad de enfoques, es importante precisar que en este caso se da al término una visión particular, al ser elegido como criterio para medir y monitorizar la gestión del patrimonio cultural inmueble. El concepto de habitabilidad se comprende, de este modo, como un mecanismo que da respuesta a las necesidades y objetivos de desarrollo social, a través de mejoras que afecten positivamente a la conservación del patrimonio.

Se entiende, en este contexto, la habitabilidad como un concepto con varias dimensiones cuyo núcleo central está constituido alrededor de la vivienda, tanto por agrupar una de las necesidades sociales más básicas relacionadas con el patrimonio -asociadas a las condiciones de vida- como por constituir uno de los ejes principales del patrimonio de las ciudades históricas. Esto cobra especial importancia por el considerable peso de la vivienda en el patrimonio inmueble de Ecuador, que representa prácticamente el 75\% del mismo.

A través de la entrevista, se contrasta la primera aproximación del enfoque elegido -la habitabilidad- y de las variables que intervienen en el mismo con personal experto en la temática y conocedor del contexto ecuatoriano, para corroborar: si dicho enfoque responde a las necesidades de gestión del patrimonio ecuatoriano; si el enfoque planteado está correctamente representado por las variables inicialmente seleccionadas a partir de la revisión bibliográfica realizada y si existen variables faltando en este primer enfoque y cuáles son éstas.

\section{III.2. Orientación de las encuestas de habitabilidad}

Como resultado del estado de cuestión sobre la habitabilidad y de la entrevista de validación se elabora una primera selección de variables que podrían ser objeto de las encuestas de habitabilidad y del resto del estudio. Se trata de una versión incipiente en la que se considera que el fenómeno de la habitabilidad está compuesto por tres dimensiones temáticas: Vivienda, Sociedad y Entorno. El motivo de separación de estos tres ámbitos se debe a la consideración de la habitabilidad como un fenómeno a analizar tanto puertas adentro de la 
vivienda con sus condiciones, como en su entorno más próximo relativo a los equipamientos y servicios cercanos a ella y la sociedad que reside en el lugar (sus principales características y condicionantes). Para cada una de estas dimensiones se definen una serie de factores o subtemáticas en las que se puede dividir la dimensión, y una serie de indicadores o conceptos para medir cada factor.

Esta primera versión de la estructura de indicadores que componen el concepto, trata de hacer una definición amplia y flexible del fenómeno de habitabilidad que pueda modelarse en los análisis siguientes, por lo que se concibe para abarcar o considerar todos los aspectos susceptibles de tener relación con el tema de estudio.

Sobre esta base se implementan unas encuestas a expertos en la temática y conocedores del contexto local de las dos ciudades analizadas, lo que sirve para orientar los análisis intraurbanos que se realizan para cada una de las dos ciudades.

\section{III.3. El papel de la cartografía estadística}

La relación entre la Estadística y la Cartografía alcanza su exponente más representativo en la cartografía estadística, entendida como "una rama de la cartografía temática que sirve para plasmar sobre un mapa información estadística referida a unidades geográficas predefinidas (áreas, puntos o líneas)” (Reques, 2002: 176). Esta disciplina se centra en analizar y tratar la información estadística y en representarla en un mapa de la forma más adecuada posible y su reto consiste en alcanzar resultados sintéticos, sencillos de entender e interpretar (Reques, 2006).

En este estudio, los objetivos principales de la aplicación de este tipo de análisis consisten en: identificar patrones espaciales de las dimensiones y aspectos que componen el concepto de habitabilidad para sobre esta base explicar el comportamiento interno de las ciudades analizada; identificar los contrastes espaciales en las condiciones de habitabilidad dentro de una misma ciudad; detectar las desigualdades sociales y de conservación que inciden en la habitabilidad tal y como se considera en esta investigación; analizar la relación que existe entre la edificación y el espacio (cómo un espacio bueno -de calidad- sube el valor y calidad de la edificación y viceversa) y analizar si existe diferencia entre el espacio urbano planificado y no planificado.

Como resultado tras la implementación de las encuestas, se realiza una nueva estructura de las dimensiones y variables que componen el concepto de habitabilidad y que servirán como estructura para la elaboración de un análisis intraurbano mediante cartografía estadística. Esta selección consta de tres dimensiones evolucionadas con respecto a los análisis anteriores (estado de la cuestión y entrevista de validación), que son: 1) Población: evolución y estructura, 2) Condicionantes sociales del habitar, y 3) Condiciones de la vivienda y del entorno.

Un aspecto relevante a considerar es que esta estructura se basa ya en datos existentes; es decir, se adaptan los conceptos identificados a datos disponibles, principalmente en el Censo de Población y Viviendas. De este modo, se avanza en la precisión de los aspectos que comprenden el fenómeno de la habitabilidad ajustados a los datos disponibles, lo que contempla ya la importancia de que el resultado del sistema sea medible. 


\section{III.4. La estadística multivariante como integración y estructuración final del sistema}

La estadística puede considerarse como "la ciencia de las regularidades que se observan en conjuntos de fenómenos" o como "la colección de métodos científicos que permiten el análisis e interpretación de la información numérica” (García, 2004: 23). Existen diferentes tipos de análisis estadístico en función de su número de variables analizadas, su carácter exploratorio o confirmatorio, su naturaleza cuantitativa o cualitativa y en función de su objetivo causal, de cambio, de decisiones, de grupos o de sistemas (Sierra, 2001: 461).

En este caso se aplican análisis tanto univariados, como bivariados y multivariados con carácter exploratorio. Con respecto a estos análisis, cabe destacar las técnicas de análisis multivariado empleadas, el análisis factorial de componentes principales y el análisis clúster, cuya aplicación conjunta se denomina también análisis ecológico-factorial.

En esta investigación el empleo de diferentes técnicas estadísticas responde a los siguientes objetivos, que son: detectar las variables redundantes, descartar las variables que no presentan variabilidad, seleccionar las variables que representan aspectos negativos de la habitabilidad y descartar las que representan aspectos positivos, analizar la interrelación entre las variables seleccionadas, definir la estructura subyacente al concepto de habitabilidad y sintetizar las variables analizadas en conceptos o factores.

Como resultado de la aplicación de estos análisis se logra una estructura de indicadores para la medición de la habitabilidad en cada ciudad compuesta de factores que agrupan indicadores y grupos o clusters que determinan clasifican las secciones censales de cada ciudad en función de sus problemáticas relativas a la habitabilidad.

\section{ANÁLISIS DE LAS CIUDADES PATRIMONIALES DE ECUADOR: LOS CASOS DE SAN GABRIEL Y SANGOLQUÍ}

Este estudio se enmarca en un contexto de predisposición política ${ }^{4}$, disponibilidad de medios financieros ${ }^{5}$ y comienzo de un proceso de descentralización en el que se inician diferentes acciones ordenadas, dirigidas por el Ministerio Coordinador de Patrimonio y el Instituto Nacional de Patrimonio para poner en valor el patrimonio cultural de Ecuador y capacitar a las municipalidades para que puedan asumir las competencias de tutela y gestión del patrimonio. Las principales acciones a nivel nacional en este sentido son la creación de la Red de Ciudades Patrimoniales y el lanzamiento del proyecto "Ciudades patrimoniales" para articular y formalizar la creación de la red, así como formar a las municipalidades involucradas en la misma.

La justificación de creación de la red viene asociada a la necesidad de mantener los centros históricos y bienes patrimoniales y a realizar una gestión en beneficio de los habitantes

4 En esta época se generan una serie de instrumentos políticos que apuestan por un cambio de modelo y de organización del país y que introducen el patrimonio en la agenda política. Los más importantes son; la Constitución 2008, el Plan Nacional del Buen Vivir 2009-2013 y el Código orgánico de organización territorial, autonomía y descentralización (COOTAD).

5 Una medida relevante a considerar debido a su influencia sobre la conservación del patrimonio fue el Decreto de Emergencia del Patrimonio Cultural que supuso el desembolso de 37 millones de dólares para abordar el rescate de la memoria social y el patrimonio, un sector nunca antes abordado por el gobierno. 
de las ciudades, con el objetivo de enfrentar los siguientes problemas (Ministerio Coordinador de Patrimonio Cultural de Ecuador, 2008: 1): la existencia de una estructura urbana no diseñada para las exigencias y demandas actuales, la degradación ambiental y del paisaje cultural, los bajos niveles de habitabilidad provocados por las intervenciones modernas y, en general, el conjunto de problemas que produce el enfrentar una configuración del pasado con la vida contemporánea.

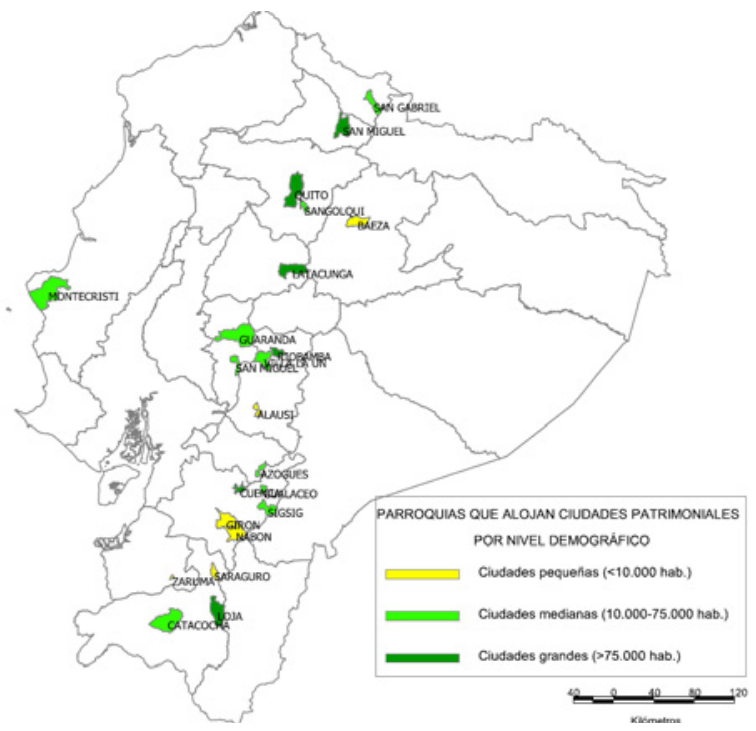

Fuentes: [Estadísticas] INEC, Censo de Población y viviendas 2001. [Cartográficas] INEC, Cartografía por secciones censales 2001. Mapa Nacional por provincias, actualizado en 2009. Elaboración propia.

De las 22 ciudades patrimonio nacional (véase Figura 4), en este estudio se presta especial atención a las de mediano tamaño (entre 10.000 y 75.000 habitantes), lo que permite discriminar los casos inferiores a los 10.000 habitantes por falta de entidad suficiente de gestión, y los casos por encima de los 75.000 habitantes, dado que éstos disponen ya de avanzados mecanismos de gestión del patrimonio. De este grupo de ciudades se escogen para este análisis las ciudades de San Gabriel y Sangolquí debido a la particularidad de haber comenzado al mismo tiempo el proceso de institucionalización de la gestión de su patrimonio impulsado por el proyecto $\mathrm{FOCAD}^{6}$, que enmarca este estudio.

El análisis se realiza a escala de sector censal (unidad mínima a la que se dispone de información estadística) y se corresponde con datos de 2001, últimos disponibles a escala intraurbana.

6 San Gabriel constituye el caso piloto oficial del proyecto FOCAD. Sangolquí comienza el proceso de manera pareja a San Gabriel y siguiendo la misma trayectoria, de la mano del Instituto de Patrimonio Cultural. 
Tabla 2

TABLA RESUMEN DE LAS VARIABLES ANALIZADAS PARA LAS CIUDADES DE SAN GABRIEL (SG) Y SANGOLQUÍ (SQ)

\begin{tabular}{|c|c|c|c|c|}
\hline Dimensión & Eje & Objetivo a medir & Variable & Ciudad \\
\hline \multirow{13}{*}{ 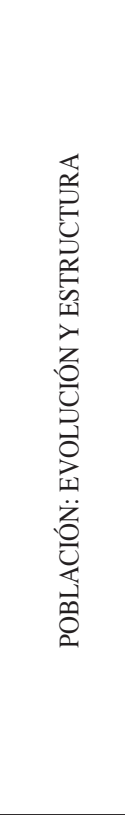 } & \multirow{2}{*}{ 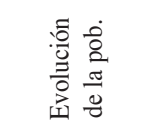 } & Crecimiento interanual & Diferencia de densidades focales entre 1990 y $2001 *$ & SG \\
\hline & & Tendencia demográfica & Índice de tendencia & Ambas \\
\hline & \multirow{5}{*}{ 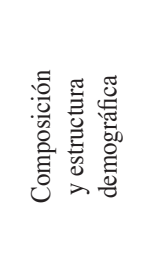 } & Perfil demográfico & $\begin{array}{l}\text { Tipología derivada de la composición según grandes } \\
\text { grupos de edad }\end{array}$ & Ambas \\
\hline & & Autoctonía & Porcentaje de población nacida en la parroquia & Ambas \\
\hline & & Población quiteña & Porcentaje de población nacida en Quito* & SQ \\
\hline & & Inmigración extranjera & $\begin{array}{l}\text { Porcentaje de población inmigrante de origen } \\
\text { extranjero }\end{array}$ & Ambas \\
\hline & & Migración & Porcentaje de viviendas con personas ausentes & Ambas \\
\hline & \multirow{6}{*}{ 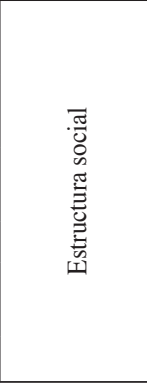 } & Perfil económico & $\begin{array}{l}\text { Tipología derivada de la composición de la } \\
\text { población según sectores económicos }\end{array}$ & Ambas \\
\hline & & Estatus socioeconómico & $\begin{array}{l}\text { Tipología derivada de la composición de la } \\
\text { población según la clasificación ocupacional }\end{array}$ & Ambas \\
\hline & & $\begin{array}{l}\text { Población con estudios } \\
\text { superiores }\end{array}$ & $\begin{array}{l}\text { Porcentaje de población con estudios superiores o } \\
\text { postgrado }\end{array}$ & Ambas \\
\hline & & Población sin estudios & Porcentaje de población sin estudios & Ambas \\
\hline & & Tenencia insegura & $\begin{array}{l}\text { Porcentaje de viviendas cuyos habitantes no son } \\
\text { propietarios }\end{array}$ & Ambas \\
\hline & & Hacinamiento del hogar & Media de ocupantes por dormitorio & Ambas \\
\hline \multirow{4}{*}{ 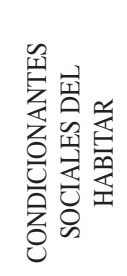 } & \multirow{4}{*}{$\begin{array}{l}\text { 苟 } \\
0 \\
0 \\
0 \\
0 \\
0 \\
0 \\
0 \\
0 \\
0 \\
0 \\
0 \\
0\end{array}$} & Fenómeno de conventillos & Porcentaje de hogares con servicio comunitario & Ambas \\
\hline & & $\begin{array}{l}\text { Carencia de servicio } \\
\text { higiénico }\end{array}$ & Porcentaje de hogares sin servicio higiénico & Ambas \\
\hline & & $\begin{array}{l}\text { Carencia de cuarto para } \\
\text { cocinar }\end{array}$ & Porcentaje de hogares sin cuarto para cocinar & Ambas \\
\hline & & Carencia de ducha & Porcentaje de hogares que no disponen de ducha & Ambas \\
\hline \multirow{7}{*}{ 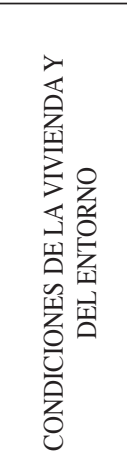 } & \multirow{3}{*}{$\frac{\mathscr{d}}{\stackrel{0}{0}} \frac{\pi}{\frac{\pi}{0}}$} & Hacinamiento de la vivienda & Porcentaje de viviendas con dos o más hogares & Ambas \\
\hline & & $\begin{array}{l}\text { Carencia de servicio de } \\
\text { abastecimiento de aguas }\end{array}$ & $\begin{array}{l}\text { Porcentaje de viviendas que no reciben } \\
\text { abastecimiento de aguas por tubería }\end{array}$ & Ambas \\
\hline & & $\begin{array}{l}\text { Carencia de servicio de } \\
\text { electricidad }\end{array}$ & Porcentaje de viviendas sin electricidad & Ambas \\
\hline & \multirow{2}{*}{ 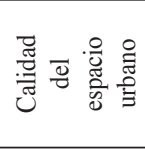 } & $\begin{array}{l}\text { Impacto visual en la fachada } \\
\text { de las viviendas }\end{array}$ & $\begin{array}{l}\text { Porcentaje de viviendas con abastecimiento de aguas } \\
\text { mediante tubería por fuera del edificio }\end{array}$ & Ambas \\
\hline & & $\begin{array}{l}\text { Riesgo de insalubridad por } \\
\text { eliminación de aguas }\end{array}$ & $\begin{array}{l}\text { Porcentaje de viviendas con eliminación de aguas } \\
\text { por pozo ciego }\end{array}$ & Ambas \\
\hline & \multirow[b]{2}{*}{$\begin{array}{l}\stackrel{0}{0} \\
\text { 总 } \\
\text { 至 }\end{array}$} & Crecimiento urbano & Porcentaje de vivienda en construcción & Ambas \\
\hline & & Antigüedad de las viviendas & Año de ocupación del espacio urbano* & SG \\
\hline
\end{tabular}

Fuente: elaboración propia. Nota: Con * se marcan aquellas variables solo analizadas para una de las ciudades. 
Como parte de este análisis, basado principalmente en fuentes estadísticas, se plantean tres dimensiones a analizar (como resultado de la estructura de habitabilidad que se menciona en el apartado 3.4): la población, los condicionantes sociales del habitar y las condiciones de la vivienda y del entorno. Cada una de estas dimensiones está compuesta por grandes bloques temáticos también llamados ejes, integrados a su vez por una serie de variables a medir, que permiten analizar y comprender los ejes bien teniendo en cuenta los diferentes fenómenos o hechos por los que están influidos, o bien matizando los hechos más relevantes de cada eje. En la Tabla 2 se presentó un cuadro resumen de las variables analizadas para cada ciudad mediante cartografía estadística, que se estructuran en: dimensiones-ejes-objetivos a medir-variables consideradas.

\section{IV.1. Análisis de la habitabilidad de la ciudad patrimonial de San Gabriel}

San Gabriel es una ciudad situada al Norte del país, cercana a la frontera con Colombia y ubicada dentro de la provincia del Carchi. Esta ciudad, de 19.230 habitantes según el censo de 2001, sufrió un leve descenso de población entre 1990 y 2001 (de un -0,68\%), pero asciende un 8,9\% entre 2001 y 2010 según las cifras del último censo publicado. Esta ciudad mantiene una estrecha relación con Colombia, con la que comparte orígenes culturales, debido a su relación fronteriza. La ciudad, está limitada por su orografía (pendientes entre el 30 y el 50\%), el río al Oeste y por la carretera Panamericana al Este, por lo que se desarrolla en su origen longitudinalmente (ver Figura 6) y crece posteriormente hacia el Este.

La ciudad de San Gabriel, antiguamente conocida como Tusa, sufre una casi completa destrucción con el terremoto de Ibarra en 1868. Este hecho, conlleva una reconstrucción de la ciudad en base al primer plano de la misma utilizado por los españoles, con forma de cuadricula o damero.

Debido a esto, la ocupación de suelo urbano de la ciudad se designa "hasta 1869" como intervalo de fechas más antiguo. El área de la ciudad cuya ocupación se corresponde con dicho intervalo, es la que se encuentra considerada como centro histórico. En el caso del primer anillo éste corresponde en su totalidad a este periodo, mientras que en el segundo anillo se mezclan áreas de ocupación de 1960 y 1970. En cualquier caso, las áreas más antiguas de la ciudad se encuentran comprendidas en el primer y segundo anillo del centro histórico.

Figura 6

FOTOGRAFIAS ILUSTRATIVAS DE LAESTRUCTURA URBANA DE SAN GABRIEL
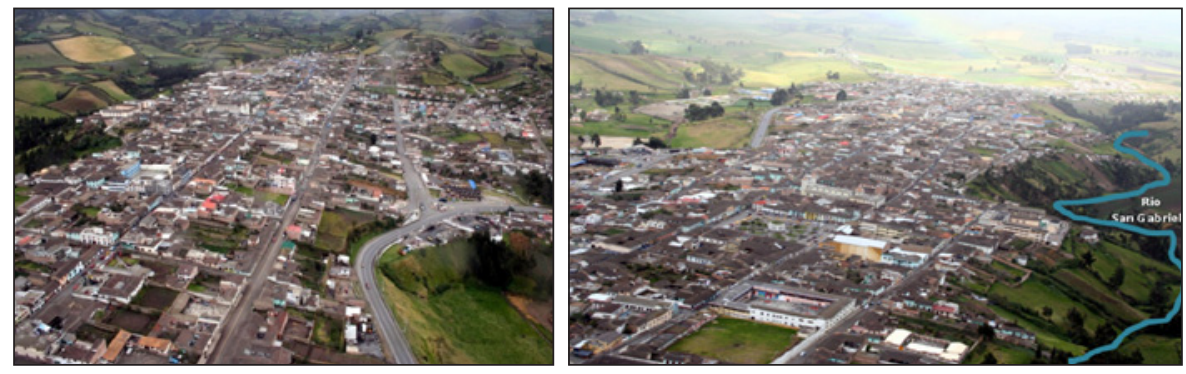

Fuente: Gobierno Municipal de Montúfar. 
El análisis estadístico-cartográfico realizado permite diferenciar una zona edificada más consolidada, con menor porcentaje de nueva construcción de viviendas, y a su vez compuesta por las construcciones más antiguas de la ciudad. Esta zona tiene una ubicación en casi toda el área del centro histórico y su espacio colindante (especialmente la ubicada en el Este). Esta área presenta unas características comunes en una gran parte de las variables analizadas, que la diferencian del resto de la ciudad, como por ejemplo: una población mayoritariamente de estatus alto o medio-alto, con alto porcentaje de población con estudios superiores, un perfil de población predominantemente dedicada al sector terciario, con menores afecciones en variables relativas a las condiciones de vida en el hogar como el índice de hacinamiento del hogar, los hogares sin ducha, sin servicio higiénico o sin cuarto para cocinar. Esto no es igual para la subdivisión de viviendas (o fenómeno de conventillos), dónde esta área es una de las más afectadas, probablemente debido a que al tratarse de viviendas más antiguas, éstas son de mayor tamaño y su subdivisión es más factible. Presenta también menores afecciones en relación a las condiciones e instalaciones de las viviendas como el abastecimiento de aguas o la disponibilidad de electricidad. Asimismo, es una de las zonas con menor riesgo de insalubridad por eliminación de aguas.

En contraposición a esta área, se podría plantear del área más periférica de la ciudad, que dividimos en dos zonas: la periferia Norte-Este y la Oeste o zona colindante al río San Gabriel. La principal diferencia entre estas dos áreas, es que la colindante al río presenta una zona más consolidada en cuanto a edificación y limitada en su crecimiento por el río y la orografía del terreno, mientras que la periférica Norte y sobretodo Este, se encuentra en vías de crecimiento, y constituye la zona de actual desarrollo y crecimiento de la ciudad.

El área colindante al río se encuentra asentada entre el centro histórico y una fuerte pendiente que desciende hacia el río. Esta presenta similitudes con el centro histórico en muchas variables debido a su cercanía y debido a que alberga también viviendas de entre 1869 y 1970 . Esta zona presenta sin embargo mayores deficiencias en sus viviendas, la convivencia de sus hogares o la calidad de su espacio urbano con respecto al centro histórico.

El área periférica Norte y Este, especialmente el área Este, es la que alberga la edificación más moderna y en la que se dan las mayores proporciones de crecimiento urbano. Se pueden resaltar los siguientes aspectos sobre esta área: su población es de estatus predominantemente bajo (salvo excepciones), dedicada principalmente a los sectores primario y terciario, con los mayores porcentajes de población sin estudios y los menores porcentajes de población con estudios superiores. Presentan algunos de los mayores porcentajes de población inmigrante extranjera, sobre todo en las secciones más al Sur. Con respecto a las condiciones de vida en los hogares destacan en el hacinamiento de sus hogares, en albergar secciones con los mayores porcentajes de hogares sin servicio higiénico, sin ducha o sin cuarto para cocinar. Salvo dos secciones, no presentan destacados problemas en la subdivisión de viviendas. Presenta considerables afecciones (aunque no de manera homogénea) en relación a las condiciones e instalaciones de las viviendas como el abastecimiento de aguas o la disponibilidad de electricidad. Asimismo, es una de las zonas con menor riesgo de insalubridad por eliminación de aguas. 
Figura 7

MAPAS DE PERFIL ECONÓMICO Y ESTATUS SOCIO-ECONÓMICO
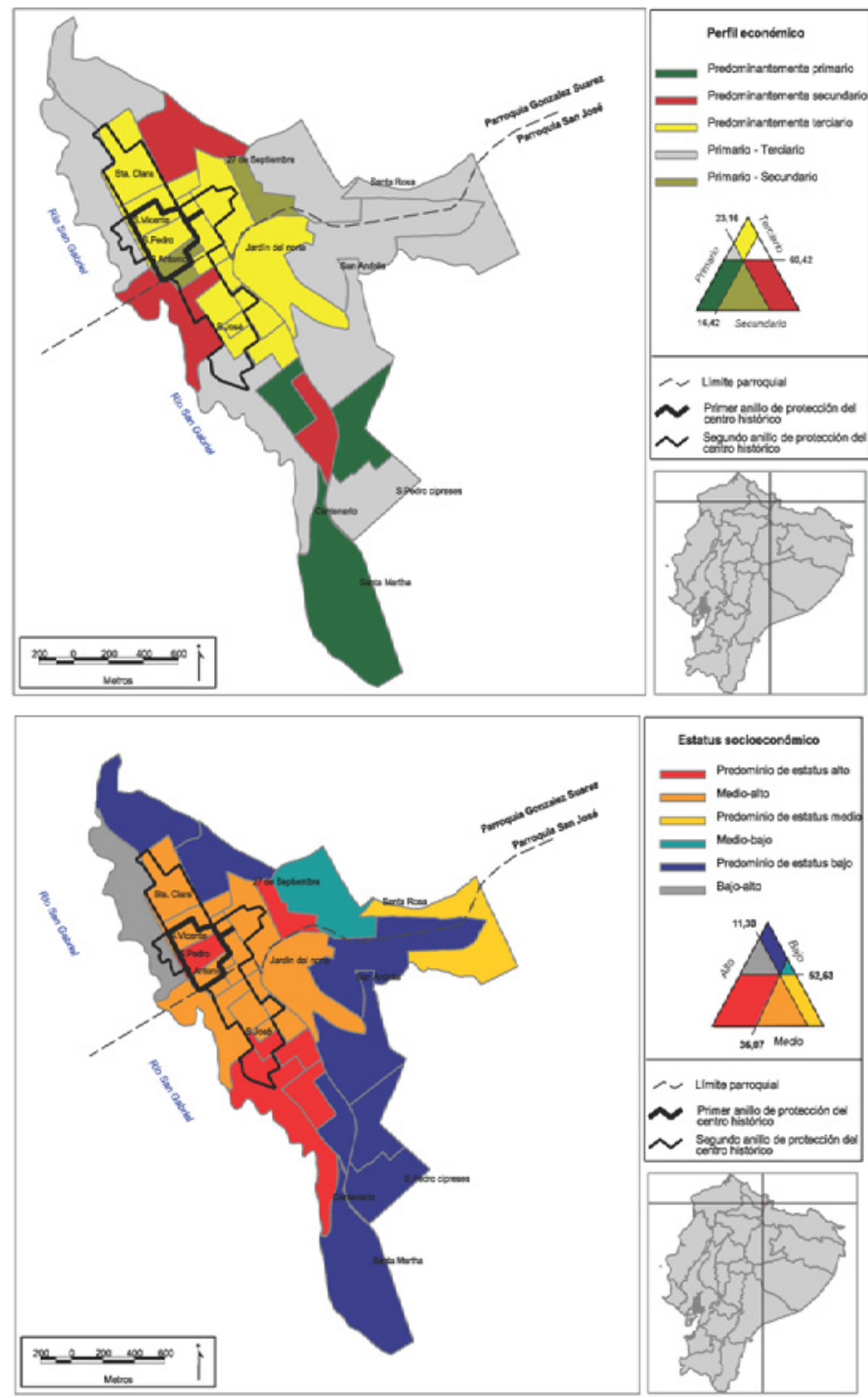

Fuentes: [Estadísticas] INEC, Censo de Población y viviendas 2001. [Cartográficas] INEC, Cartografía por secciones censales 2001. Mapa Nacional por provincias, actualizado en 2009. Elaboración propia.

\section{IV.2. Análisis de la habitabilidad de la ciudad patrimonial Sangolquí}

Sangolquí es una ciudad ubicada en la provincia de Pichincha, considerada parte de la gran conurbación que forma el área metropolitana de Quito, ciudad con la que mantiene vín- 
culos geográficos, históricos y poblacionales. Esta ciudad, que contaba con 43.397 habitantes en 1990 sufre un crecimiento del $44 \%$ hasta 2001 alcanzando la cifra de 62.562 habitantes y de casi un $30 \%$ entre 2001 y 2010 culminando con 81.140 habitantes. El motivo de este crecimiento se debe a que Sangolquí forma parte de la conurbación del distrito metropolitano de Quito, ejerciendo un rol de ciudad dormitorio o área de expansión de la capital.

Figura 8

MAPA DE PERFIL ECONÓMICO

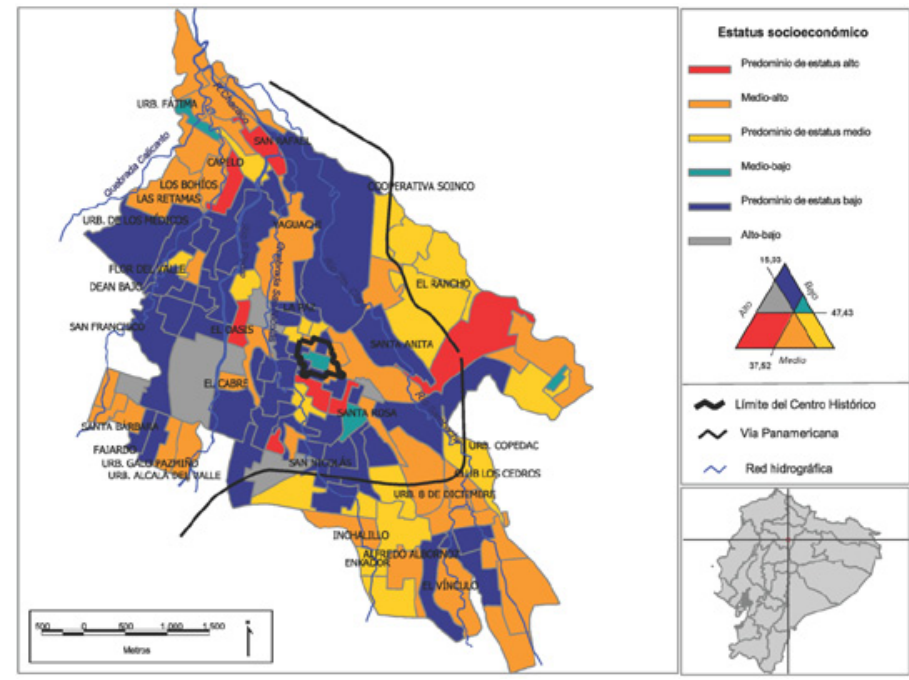

Fuentes: [Estadísticas] INEC, Censo de Población y viviendas 2001. [Cartográficas] INEC, Cartografía por secciones censales 2001. Mapa Nacional por provincias, actualizado en 2009. Instituto Geográfico Militar, Ríos de Sangolquí 2011. Vías de Sangolquí 2011 Nombres áreas de Sangolquí 2011. Elaboración propia.

En el caso de Sangolquí, a diferencia de San Gabriel, no existe un patrón espacial tan homogéneo sobre las diferencias de habitabilidad entre las secciones que componen la ciudad; sin embargo, sí existe una clara relación entre las variables de población que determinan las condiciones de inhabitabilidad zonificando estas la ciudad en áreas bien diferenciadas.

Entre las variables consideradas destaca la de perfil económico de la población, que aporta un patrón espacial que sirve para zonificar y distinguir las diferentes categorías de habitabilidad de la ciudad. La población dedicada a los sectores primario y secundario está claramente asociada a peores condiciones de habitabilidad y a población autóctona de la ciudad, mientras que, por el contrario, la población dedicada al sector terciario está asociada a un mayor estatus, mejores condiciones de habitabilidad y está representada por la población de origen quiteño. La única salvedad a este caso son las secciones de los barrios Rancho, Santa Anita y Santa Rosa que, si bien en su mayoría tienen un perfil predominantemente terciario, presentan graves problemas de habitabilidad. 
En cualquier caso, lo que es clave para identificar las zonas de mejor habitabilidad es el asentamiento de la población quiteña. Esta población proveniente de la capital cuenta con mayores recursos y dónde se ubica se encuentran las mejores condiciones de habitabilidad de la ciudad. Por el contrario, las secciones donde más población autóctona se asienta están caracterizadas por peores condiciones de habitabilidad y por población con menores recursos y posibilidades (estatus bajo, sin estudios, etc.).

Figura 9

MAPAS DE POBLACIÓN ORIGINARIA DE QUITO Y ESTATUS SOCIO-ECONÓMICO
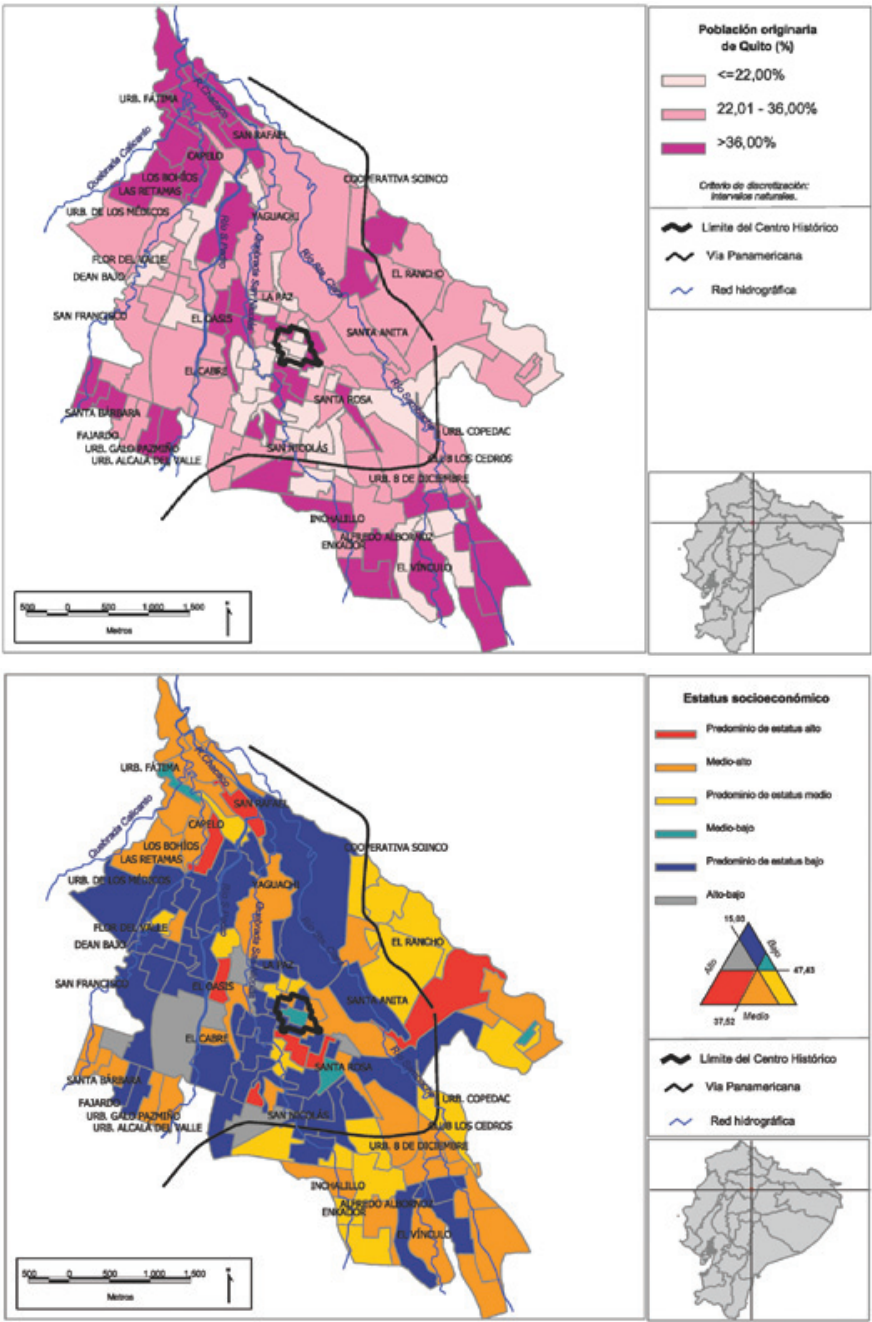

Fuentes: [Estadísticas] INEC, Censo de Población y viviendas 2001. [Cartográficas] INEC, Cartografía por secciones censales 2001. Mapa Nacional por provincias, actualizado en 2009. Instituto Geográfico Militar, Ríos de Sangolquí 2011. Vías de Sangolquí 2011 Nombres áreas de Sangolquí 2011. Elaboración propia. 


\section{LA INTEGRACIÓN DE TÉCNICAS DE ANÁLISIS COMO INSTRUMENTO DE DIAGNÓSTICO DE LA HABITABILIDAD}

Con la finalidad de profundizar en el análisis realizado anteriormente se plantea la incorporación de técnicas estadísticas de carácter multivariado que permitan avanzar en la obtención de resultados sintéticos para los ámbitos considerados. Con respecto a estos análisis, cabe destacar las técnicas de análisis multivariado empleadas, el análisis factorial de componentes principales y el análisis de conglomerados o cluster.

Dado el reducido número de casos (secciones) de los que se compone la ciudad de San Gabriel no es posible obtener resultados significativos de la misma, por lo que se realiza en primer lugar el análisis de la ciudad de Sangolquí para emplear en un segundo lugar el de la ciudad de San Gabriel como contraste o validación de los resultados obtenidos. Con este objetivo se plantean a continuación algunas conclusiones de contraste entre ambos casos.

Si bien a través de los análisis bivariados y multivariados coinciden en ambas ciudades las variables asociadas al fenómeno de inhabitabilidad existen algunas excepciones como es la variable Población nacida en la parroquia (de San Gabriel o Sangolquí, según la ciudad analizada), que en el caso de Sangolquí está asociada a condiciones de inhabitabilidad, mientras que en el caso de San Gabriel está asociada a condiciones de habitabilidad. Esto muestra fenómenos diferentes propios de la dinámica actual de dichas ciudades, en el caso de Sangolquí la población autóctona está siendo desplazada por la población quiteña, con mayor poder adquisitivo, lo que hace a la población de autóctona más propensa a tener problemas de habitabilidad.

Otro ejemplo es la variable Viviendas de tipo departamento, que en Sangolquí se asocian a condiciones positivas de habitabilidad, mientras que en San Gabriel se asocian a condiciones de inhabitabilidad.

Como resultado del análisis factorial se seleccionan tres factores para cada ciudad compuestos por los indicadores que se pueden ver en la Tabla 3 .

Considerando los resultados de los análisis factoriales realizados para cada una de las ciudades y su composición por variables, se puede señalar que:

Ambas ciudades presentan un factor denominado Infravivienda, que mantiene como variables comunes cocina con carbón y carencia de ducha. En el caso de Sangolquí este tiene dos variables (sector secundario y población originaria de Sangolquí) que se han descartado para San Gabriel, y en el caso de San Gabriel, el resto de variables que componían el factor en Sangolquí pasan a subdividirse en dos factores (junto con otras variables adicionales):

- Infravivienda: más centrado en la vivienda, que incluye además de cocina con carbón y carencia de ducha; eliminación de aguas por pozo ciego o pozo séptico, que representa ella sola un único factor en el caso de Sangolquí y carencia de servicio higiénico que si bien se desestima en el factorial de Sangolquí, se vuelve a incluir en su análisis cluster.

- Estatus bajo: más centrado en la población residente y que incluye las variables población dedicada al sector primario, población sin estudios y suelo de tierra, que formaban parte del factor Infravivienda en Sangolquí y las agrupa junto a otras variables consideradas en el factor Hacinamiento de Sangolquí (estatus bajo y ocupantes por dormitorio) y con la variable viviendas de tipo mediagua, que en el caso de Sangolquí había sido descartada. 
Tabla 3

TABLA COMPARATIVA DE LOS FACTORES OBTENIDOS PARA CADA CIUDAD Y LAS VARIABLES QUE LOS COMPONEN

\begin{tabular}{|c|c|c|c|c|}
\hline \multirow{2}{*}{$\begin{array}{l}N^{o} \text { de } \\
\text { Factor }\end{array}$} & \multicolumn{2}{|l|}{ Sangolquí } & \multicolumn{2}{|l|}{ San Gabriel } \\
\hline & Denominación & $\begin{array}{l}\text { Variables de las que se } \\
\text { compone }\end{array}$ & Denominación & $\begin{array}{l}\text { Variables de las que se } \\
\text { compone }\end{array}$ \\
\hline $\mathrm{F} 1$ & $\begin{array}{l}\text { Infravivienda } \\
\text { (explica un } \\
32,51 \% \text { de la } \\
\text { varianza) }\end{array}$ & $\begin{array}{l}\text { Porcentaje de personas que } \\
\text { trabajan en el sector primario } \\
\text { Porcentaje de población sin } \\
\text { estudios } \\
\text { Porcentaje de hogares que } \\
\text { cocinan con leña o carbón } \\
\text { Porcentaje de viviendas con } \\
\text { suelo de tierra } \\
\text { Porcentaje de hogares sin } \\
\text { ducha } \\
\text { Porcentaje de personas } \\
\text { que trabajan en el sector } \\
\text { secundario* } \\
\text { Porcentaje de población } \\
\text { nacida en la parroquia de } \\
\text { Sangolquí* }\end{array}$ & $\begin{array}{l}\text { Estatus bajo } \\
\text { (explica un } \\
35,55 \% \text { de la } \\
\text { varianza) }\end{array}$ & $\begin{array}{l}\text { Porcentaje de personas de } \\
\text { estatus bajo } \\
\text { Porcentaje de población sin } \\
\text { estudios } \\
\text { Porcentaje de viviendas de } \\
\text { tipo mediagua* } \\
\text { Media de ocupantes por } \\
\text { dormitorio } \\
\text { Porcentaje de personas } \\
\text { que trabajan en el sector } \\
\text { primario } \\
\text { Porcentaje de viviendas con } \\
\text { suelo de tierra }\end{array}$ \\
\hline $\mathrm{F} 2$ & $\begin{array}{l}\text { Hacinamiento } \\
\text { (explica un } \\
27,65 \% \text { de la } \\
\text { varianza) }\end{array}$ & $\begin{array}{l}\text { Porcentaje de viviendas de } \\
\text { tipo cuartería* } \\
\text { Porcentaje de hogares con } \\
\text { servicio compartido } \\
\text { Media de ocupantes por } \\
\text { dormitorio } \\
\text { Porcentaje de hogares sin } \\
\text { cuarto para cocinar* } \\
\text { Porcentaje de personas de } \\
\text { estatus bajo } \\
\text { Porcentaje de viviendas con } \\
\text { paredes de adobe* }\end{array}$ & $\begin{array}{l}\text { Infravivienda } \\
\text { (explica un } \\
30,31 \% \text { de la } \\
\text { varianza) }\end{array}$ & $\begin{array}{l}\text { Porcentaje de hogares sin } \\
\text { ducha } \\
\text { Porcentaje de hogares sin } \\
\text { servicio higiénico* } \\
\text { Porcentaje de viviendas con } \\
\text { eliminación de aguas por } \\
\text { pozo ciego o pozo séptico } \\
\text { Porcentaje de hogares que } \\
\text { cocinan con leña o carbón }\end{array}$ \\
\hline F3 & $\begin{array}{l}\text { Riesgo de } \\
\text { insalubridad } \\
\text { (explica un } \\
9,13 \% \text { de la } \\
\text { varianza) }\end{array}$ & $\begin{array}{l}\text { Porcentaje de viviendas con } \\
\text { eliminación de aguas por } \\
\text { pozo ciego o pozo séptico }\end{array}$ & $\begin{array}{l}\text { Servicio } \\
\text { comunitario } \\
\text { (explica un } \\
12,32 \% \text { de la } \\
\text { varianza) }\end{array}$ & $\begin{array}{l}\text { Porcentaje de hogares con } \\
\text { servicio compartido } \\
\text { Porcentaje de viviendas de } \\
\text { tipo departamento* }\end{array}$ \\
\hline
\end{tabular}

Fuente: elaboración propia. Con * se presentan las variables no comunes a ambos casos.

El factor Hacinamiento que presenta Sangolquí, que presenta como variables propias que han sido descartadas en San Gabriel viviendas tipo cuartería, carencia de cuarto para cocinar y paredes de adobe, se divide en el caso de San Gabriel en dos factores: Estatus bajo, ya mencionado y; Servicio comunitario: que junto con la variable servicio comunitario presenta la variable vivienda tipo departamento, descartada para el caso de Sangolquí.

El factor Riesgo de insalubridad de Sangolquí, compuesto exclusivamente por la variable eliminación de aguas por pozo ciego y pozo séptico pasa a formar parte del factor Infravivienda en el caso de San Gabriel. 


\begin{tabular}{|c|c|c|}
\hline Grupo & Sangolquí & San Gabriel \\
\hline 1 & $\begin{array}{l}\text { Secciones con carencia de servicio } \\
\text { higiénico e infravivienda (agrupa un } \\
25 \% \text { de los casos): } \\
\text { - Porcentaje de hogares sin servicio } \\
\text { higiénico } \\
\text { - En menor medida los factores } 1 \text { y } 2\end{array}$ & $\begin{array}{l}\text { Secciones afectadas por infravivienda } \\
\text { (agrupa un } 4 \% \text { de los casos): } \\
\text { - Factor } 2 \text { Infravivienda }\end{array}$ \\
\hline 2 & $\begin{array}{l}\text { Secciones con riesgo de insalubridad } \\
\text { y baja calidad de vivienda (agrupa un } \\
\mathbf{1 1 \%} \text { de los casos): } \\
\text { - Factor } 3 \text { Insalubridad } \\
\text { - En menor medida factores } 1,2 \text { y } \\
\text { Porcentaje de hogares sin servicio } \\
\text { higiénico }\end{array}$ & $\begin{array}{l}\text { Secciones con población de estatus bajo y } \\
\text { bajas condiciones de vida (agrupa un } \mathbf{2 4 \%} \\
\text { de los casos): } \\
\text { - Factor } 1 \text { Estatus bajo } \\
\text { - Factores } 2 \text { y } 3 \text { en menor medida }\end{array}$ \\
\hline 3 & $\begin{array}{l}\text { Secciones con riesgo de hacinamiento } \\
\text { (agrupa un } 32 \% \text { de los casos): } \\
\text { - Factor } 2 \text { Hacinamiento }\end{array}$ & $\begin{array}{l}\text { Secciones con hogares que comparten } \\
\text { servicio higiénico (agrupa un } 32 \% \text { de los } \\
\text { casos): } \\
\text { - Factor } 3 \text { Servicio Comunitario }\end{array}$ \\
\hline
\end{tabular}

Fuente: elaboración propia.

Los resultados de los análisis cluster dan en ambos casos cuatro grupos o tipologías de caso, entre los cuales ambos coinciden en el grupo Secciones Habitables, compuesto por puntuaciones negativas en todos los factores de vulnerabilidad analizados.

En el caso de los grupos restantes, la composición y resultado para ambas ciudades se muestra a continuación en la Tabla 4 por orden de vulnerabilidad.

Estos resultados, si bien son algo dispares entre ciudades por las diferencias entre los factores resultantes para cada ciudad, mantienen características muy similares centradas en las siguientes temáticas:

- Carencias de infraestructuras o servicios básicos de la vivienda: como por ejemplo servicio higiénico, ducha, cuarto para cocinar, etc.

- Viviendas realizadas con materiales de bajo coste: paredes de adobe, suelo de tierra, etc.

- Fórmulas de convivencia que denotan hacinamiento o baja calidad de vida: ocupantes por dormitorio, servicio comunitario, etc.

- Perfil de población con bajas oportunidades o posibilidades: estatus bajo, sin estudios, dedicada al sector primario, etc.

Estas temáticas parecen constituir una estructura común que subyace en los dos casos analizados y que podría servir de orientación para el análisis de la inhabitabilidad en otras ciudades del mismo tamaño en Ecuador. 


\section{A MODO DE CONCLUSIÓN}

El proceso realizado ha servido para definir un método multiescala que permite analizar y medir el fenómeno de la habitabilidad en ciudades patrimoniales. Este método se basa en 4 fases de trabajo (1. Representación del concepto; 2. Procesado de las variables seleccionadas; 3. Construcción del sistema, y 4. Validación) y combina diferentes tipos de análisis propios del análisis geográfico y social, con un énfasis en el análisis espacial de los diferentes indicadores analizados mediante SIG y culmina con la creación de un índice de inhabitabilidad que sirve para comparar el diferente grado de habitabilidad de las diferentes áreas de una ciudad.

Es importante señalar que en el caso de la vulnerabilidad vinculada a las condiciones de habitabilidad, dependiendo del contexto y el grado de problemas elementales que haya ya resueltos, los indicadores para su medición deberán basarse en aspectos básicos, pudiendo variar desde cuestiones como las viviendas sin servicio higiénico hasta aspectos de confort percibido. En cualquier caso, los indicadores mínimos a considerar en el caso de Ecuador, comunes en ambos casos analizados y replicables a otras ciudades gracias al empleo de datos censales, son: porcentaje de población dedicada al sector primario, porcentaje de población de estatus bajo, porcentaje de población sin estudios, media de ocupantes por dormitorio, porcentaje de viviendas con suelo de tierra, porcentaje de hogares sin ducha, porcentaje de hogares con servicio compartido, porcentaje de hogares que cocinan con leña o carbón y porcentaje de viviendas con eliminación de aguas por pozo ciego o séptico. Esta selección puede variar dependiendo del contexto en el que se trabaje, y debe ser previamente analizado para realizar una buena selección de variables a analizar.

Por otro lado, con independencia de los aspectos medidos, a través de este estudio se demuestra que los datos demográficos y sociales pueden ofrecer una idea del grado de inhabitabilidad o vulnerabilidad sin necesidad de entrar propiamente en datos de vivienda. Esto puede facilitar este tipo de análisis ya que los datos más frecuentemente disponibles suelen ser los de población. De todos modos, no hay que olvidar que estos datos deben ser igualmente trabajados con conocimiento del contexto, ya que en algunas ocasiones hay colectivos de población, como la población autóctona o la inmigrante, que dependiendo del contexto son los colectivos más vulnerables o, por el contrario, los menos.

Si bien pueden incluirse indicadores adicionales que permitan ampliar, complementar o interpretar el grado de inhabitabilidad y sus causas, se considera que debe haber un núcleo formado por las variables más expresivas, y luego otros indicadores descriptivos que nos permitan complementar o interpretar: qué población vive en los casos más extremos, que otras variables reflejan sus sistema de vida y sus condiciones, como afecta o condiciona el entorno, las características, la ubicación, etc.

Finalmente, y en base a la comparativa de resultados de los análisis estadísticos, se puede concretar que en cuestiones de habitabilidad hay una serie de aspectos comunes, que independientemente de los indicadores que se empleen para medirlos, forman una estructura común al fenómeno de inhabitabilidad y que puede servir para replicar el método diseñado en ciudades latinoamericanas similares a las analizadas. Esta estructura se centra en las siguientes temáticas: carencias de infraestructuras o servicios básicos de la vivienda, viviendas realizadas con materiales de bajo coste, fórmulas de convivencia que denotan hacinamiento o baja calidad de vida y perfil de población con bajas oportunidades o posibilidades. 


\section{BIBLIOGRAFÍA}

BRIZ CARO, J. (2010): Ponencia: “Rehabilitación integral del interior de las viviendas. Las prestaciones de las viviendas. La mejora continua”. Congreso Internacional Rehabilitación y sostenibilidad. El futuro es posible. Octubre 2010.

CEPAL, UN-HABITAT, ORGANIZACIÓN PANAMERICANA DE LA SALUD (OPS). (2006): Vivienda Saludable: Reto del Milenio en los Asentamientos Precarios de América Latina y el Caribe. Guía para las Autoridades Nacionales y Locales. OPS.

DÁlENÇON, R.; JUSTINIANO, C.; MÁRQUEZ, F.; VALDERRAMA, C. (2008): Artículo "Parámetros y estándares de la habitabilidad: calidad en la vivienda, el entorno inmediato y el conjunto habitacional”, parte de la publicación Camino al bicentenario, propuestas para Chile. Concurso políticas públicas 2008. Editado por: Gobierno de Chile, Pontificia Universidad Católica de Chile, Cámara de diputados de Chile y Biblioteca del Congreso Nacional de Chile.

DOMÍNGUEZ, J.; PARREÑO, J.M.; PÉRES, T. (2006): Un sistema de información geodemográfica: vivienda y población en el barrio de Escaleritas Las Palmas de Gran Canaria. Promovido por: Ayuntamiento de las Palmas de Gran Canaria y el Fondo FEDER de la Comisión Europea. Disponible on-line en: <http://geoportal.laspalmasgc.es/sicam_ doc/geodemografia/apartado_presentacion.pdf> [Consultado en Enero 2016]

GARCIA, M. (2004): Socioestadística. Introducción a la estadística en Sociología. Alianza Editorial, ciencias sociales: Madrid.

LAUDLERO, A. (2011): Habitabilidad básica: indicador estratégico para superar la pobreza, Cuadernos de Investigación Urbanística, 76 (monográfico, 111 p.)

MINISTERIO COORDINADOR DE PATRIMONIO CULTURAL DE ECUADOR. (2008): Acta de constitución de la red de ciudades que poseen centros históricos declarados como patrimonio cultural del estado.

MINISTERIO COORDINADOR DE PATRIMONIO CULTURAL DE ECUADOR. (2008): Perfil de proyecto "Red de ciudades patrimoniales del ecuador". Disponible on-line en: $<$ www.ministeriopatrimonio.gov.ec/index.php/.../67-ciudades-patrimoniales $>$ [Consultado en Septiembre de 2010]

ORGANIZACIÓN MUNDIAL DE LA SALUD (OMS). (1990): Principios fundamentales de la vivienda. Ginebra.

ORGANIZACIÓN MUNDIAL DE LA SALUD (OMS); ORGANIZACIÓN PANAMERICANA DE LA SALUD (OPS). (2006): Estrategia de la vivienda saludable.

ORGANIZACIÓN DE NACIONES UNIDAS (1948): Declaración Universal de Derechos Humanos. Disponible on-line en: < http://www.un.org/es/documents/udhr/> [Consultado en Agosto 2013]

REQUES, P. (2002): De las cifras a los mapas: el tratamiento cartográfico de la información estadística. Publicado en: El Campo de las Ciencias y las Artes, pp. 175-203. Servicio de Estudios BBVA.

REQUES, P. (2006): Geodemografía. Fundamentos conceptuales y metodológicos. Servicio de publicaciones de la Universidad de Cantabria.

SIERRA, R. (2001): Técnicas de Investigación social TEORÍA Y EJERCICIOS. Madrid: editorial Paraninfo. Decimocuarta edición. 
SOLANAS T. (2010): Ponencia "La necesidad de un nuevo concepto de habitabilidad". Congreso Internacional Rehabilitación y sostenibilidad. El futuro es posible. Octubre 2010.

USOBIAGA, E. (2012): El uso de los SIG en el proceso de diseño de un sistema de indicadores. Actas XIII Congreso de la población española "La población en clave territorial. Procesos, estructuras y perspectivas de análisis” (Septiembre, 2012). 